\title{
PAPER
}

\section{Effects of light fingertip touch on postural responses in subjects with diabetic neuropathy}

\author{
R Dickstein, R J Peterka, F B Horak
}

J Neurol Neurosurg Psychiatry 2003;74:620-626

See end of article for authors' affiliations ......................

Correspondence to: Dr F B Horak, PT Neurological Sciences Institute of Oregon Health

\& Science University, West Campus - Bldg 1, 505 NW 185th Avenue Beaverton, OR 97006-3499, USA horakf@ohsu.edu

Received 18 March 2002 In revised form 26 July 2002

Accepted

21 November 2002

\begin{abstract}
Objectives: To investigate the potential benefits from lightly touching an external supporting device on automatic postural responses to support surface translations, in subjects with profound sensory neuropathy in the feet due to diabetes mellitus (DM-PN).

Methods: Eight subjects with DM-PN and 10 age matched controls were tested under randomly ordered conditions of no fingertip touch (NT), light touch (LT; $<1 \mathrm{~N})$, and heavy touch (HT, as needed) of a stationary touch plate, during three backward translation velocities of the support surface at 10, 20 , and $30 \mathrm{~cm} / \mathrm{s}$. Dependent variables included response latencies, CoP velocity, and the slope of the relation between centre of pressure (CoP) velocity and translation velocity as a measure of response scaling.

Results: Postural response latencies were significantly longer and scaling of initial response magnitude in proportion to translation velocity was significantly smaller in the DM-PN subjects compared to the control subjects. LT had no significant effect on response latencies of the DM-PN patients. Fingertip touch increased the slope of the scaling of postural response magnitude in both groups. However, DM-PN subjects had to use HT to improve response scaling, whereas control subjects improved scaling with LT as well as HT. LT significantly increased rightward CoP velocity towards the touch plate in all subjects.

Conclusions: LT did not reduce the latency or improve the scaling of automatic postural responses in subjects with peripheral neuropathy. The major effect of LT on the automatic postural responses of the DM-PN subjects was in increasing CoP velocity towards the side of the supporting device. HT in neuropathy subjects and LT in age matched control subjects increased the sensitivity of initial postural response scaling, suggesting that somatosensory substitution from a cane in the hand could be used to improve the magnitude of medium latency postural responses to slips and trips.
\end{abstract}

$\mathrm{T}$ he importance of somatosensory information in the feet and legs to the control of stance balance is well established. ${ }^{1-3}$ The contribution of fingertip somatosensory cues to stance stability has been described more recently. Lightly touching an external supportive device with forces that provide only somatosensory cues (under $1 \mathrm{~N}$ ), significantly attenuated postural sway of healthy individuals standing with eyes closed, similar to the reduction in sway caused by heavy touch that provides mechanical support. ${ }^{4-6}$ Furthermore, the reduction in sway due to light touch (LT) of a fingertip on a stable surface was greater than sway reduction due to vision, in both healthy subjects and in subjects with bilateral loss of vestibular function. ${ }^{7}$ Likewise, light touch cues were shown to be as effective as heavy force contact in providing stabilisation of stance in blind subjects. ${ }^{8}$ In healthy subjects, LT from a fingertip also helped to suppress vibration induced postural sway from aberrant proprioceptive information from the feet, ${ }^{10}$ thereby pointing to its potential to substitute for impaired or altered somatosensory information from the feet and legs.

Distal polyneuropathy, affecting more than $50 \%$ of diabetic patients, 112 results in impaired stance balance. Patients with somatosensory loss due to diabetic neuropathy show larger sway areas, larger sway velocities, and larger sway variance than healthy control subjects. ${ }^{13-16}$ Our recent study showed that these subjects with neuropathy could significantly reduce trunk and centre of pressure (CoP) root mean square sway with light touch as much as age matched control subjects during quiet stance, and the benefit of light touch was as much as the benefit of vision. ${ }^{17}$ Thus, despite the potential for some loss of somatosensory sensitivity of the fingers, as well as the feet, subjects with profound somatosensory loss in the feet could use sensory information from a fingertip to reduce postural instability during stance. These results are consistent with the well recognised strategy used by healthy subjects on an unstable surface or by blind or vestibular loss subjects of using touch cues for improving balance and spatial orientation.

However, most studies of the use of light touch to improve balance have been limited to its effect on sway in quiet stance. Subjects with somatosensory loss due to diabetic neuropathy also show abnormal dynamic postural stability as quantified by delayed onsets of electromyography (EMG) and CoP latencies in leg muscles to postural perturbations induced by backward surface translations. ${ }^{18}$ In addition, diabetic subjects were less able to scale the magnitude of automatic postural responses for increasing velocities of surface translations; slopes of the regression lines between the initial rate of change of active torque response and platform velocity were lower in the patients than in healthy subjects. ${ }^{18}$ Inability to effectively recover from a slip or trip may be responsible for the observation that subjects with neuropathy are about 15 times more likely to report an injury during walking or standing than healthy controls. ${ }^{13}{ }^{19}$ Poor postural responses may also explain why these patients ambulate in a more conservative pattern

Abbreviations: $A P$, anterior-posterior; CoP, centre of pressure; DM-PN, diabetes mellitus peripheral neuropathy; $E M G$, electromyography; $H T$, heavy touch; LT, light touch; MG, medial gastrocnemius; ML, medio-lateral; MP, metatarsal phalangeal; NT, no touch; VOR, vestibulo-ocular reflex 
Table 1 Results of patients' nerve conduction velocity tests

\begin{tabular}{|c|c|c|c|c|c|c|c|c|}
\hline \multirow[b]{3}{*}{ Patient } & \multicolumn{4}{|c|}{ Sensory $(\mathrm{m} / \mathrm{s})$} & \multicolumn{4}{|c|}{ Motor $(\mathrm{m} / \mathrm{s})$} \\
\hline & \multicolumn{2}{|c|}{ Med plantar } & \multicolumn{2}{|l|}{ Sural } & \multicolumn{2}{|c|}{ Peroneal } & \multicolumn{2}{|c|}{ Post tibial } \\
\hline & Right & Left & Right & Left & Right & Left & Right & Left \\
\hline$M L$ & NR & NR & NR & NR & 38.3 & 23.5 & 25 & 36 \\
\hline $\mathrm{BP}$ & NR & NR & NR & NR & 38.1 & 50.0 & 50.6 & 47.6 \\
\hline PF & NR & NR & 41.2 & 33.3 & 35.3 & 38.6 & 37.8 & 40.6 \\
\hline $\mathrm{DH}$ & NR & NR & NR & NR & 35.3 & 39.4 & 34.9 & 39.9 \\
\hline Vo & NR & NR & 42.4 & 37.8 & 35.9 & 37.1 & 40.5 & 45.5 \\
\hline WD & NR & NR & NR & NR & 33.7 & 32.4 & 33.2 & 32.1 \\
\hline NW & NR & NR & NR & NR & 36.4 & 38.3 & 40.3 & 41.6 \\
\hline RM & NR & NR & NR & NR & 34.5 & 29.5 & 35.6 & 37.5 \\
\hline
\end{tabular}

Normal lab values for peroneal nerve conduction velocity are $\geqslant 40 \mathrm{~m} / \mathrm{s}$, and for the post tibial nerve $\geqslant 45$ $\mathrm{m} / \mathrm{s}$. Normal values for the sensory nerve conduction velocities are $\geqslant 40 \mathrm{~cm} / \mathrm{s}$ with foot temperature $29-30^{\circ} \mathrm{C}$.

$N R$, no response.

than healthy subjects, as shown by smaller stride lengths, slower speed, and greater proportion of time in double stance. ${ }^{20}$

The extent to which light fingertip touch, as from a cane, can improve dynamic aspects of postural stability is unknown. If the direction and velocity of a postural perturbation can be encoded by cutaneous and proprioceptive information in the arm of a subject touching a stable surface, perhaps this sensory information can be used to trigger faster and more sensitively scaled postural responses. Postural responses in the legs in response to hand perturbations have been detected with latencies as short at $50 \mathrm{~ms} .{ }^{21}$ On the other hand, touching a stable support may assist postural responses by decreasing the necessity of quick and forceful leg postural responses. It has been shown that gripping a stable support decreases the magnitude and increases the latency of automatic postural responses in the legs to surface rotations or pelvis nudges. ${ }^{22}{ }_{23}$ Mechanical support from heavy touch on a cane has been shown to improve balance performance of diabetic subjects attempting to lift a leg on a moving support surface, but it is not known whether light touch that does not provide mechanical support could also prove useful.

In the present work, we compared the potential benefits of light and heavy fingertip touch on a stable support on shortening postural response latencies and increasing the response scaling of automatic postural responses to surface translations in subjects with neuropathy. The findings have implications for the use of touch cues or a cane for the prevention of, or improving the recovery from, stumbling. These may apply not only to subjects with diabetic neuropathy but also to other elderly subjects at high risk for falling. In elderly subject, about $50 \%$ of falls are thought to be due to a slip or trip, ${ }^{24}$ and peripheral sensory neuropathy is a major identified risk factor. ${ }^{35-27}$

\section{MATERIAL AND METHODS}

\section{Subjects}

Subjects included eight patients with diabetes mellitus (mean age 59.7 (SD 10.7) years; five men, three women) with profound sensory peripheral neuropathy (DM-PN). Only completely independent community dwellers with no functional visual or motor deficits were accepted. Ten age matched healthy volunteers meeting identical demographic characteristics (mean age 61.1 (SD 9.3) years; six men, four women) served as control subjects.

In all participants, normal function of the vestibular system was verified by sinusoidal horizontal rotation testing of the vestibulo-ocular reflex (VOR). All subjects in the patient and control groups had gain and phase measures of the VOR well within the $95 \%$ tile of normal laboratory limits (for example, for $0.5 \mathrm{~Hz}$ rotations, patient group mean VOR gain was 0.67 and control group gain was 0.55 compared to a lower $95 \%$ tile limit of normal gain of 0.45). Existence of peripheral neuropathy in the patients' lower extremities was established by electrodiagnostic testing and clinical examination of their feet. Table 1 shows results of the electrodiagnostic tests.

Clinical examination included a routine physical examination of ankle joint range of motion, strength, and Achilles tendon stretch reflex, vibration sensation threshold at the sites of the medial malleoli and the first metatarsal phalangeal (MP) joint ( $128 \mathrm{~Hz}$ tuning fork), and proprioceptive sensation at the ankles and the first MP joints.

Cutaneous sensation was assessed by Semmes-Weinstein monofilaments ${ }^{28}$ at three dorsal and two plantar foot zones supplied by five cutaneous branches (fig 1 ). The testing protocol followed the interval comparison method. ${ }^{29}$ The highest testable threshold by the Semmes-Weinstein filaments is with a 6.65 filament (applying force of $279.4 \mathrm{~g}$ ), so a score of 7 was applied to foot zones in which patients were unresponsive to this filament. A comparison between Semmes-Weinstein scores of the patients and the controls pointed to substantial sensory loss in the patients, which was more pronounced on the plantar than on the dorsal aspect of the feet (fig 1).

Somatosensory sensation of the right index finger was measured by clinical testing of joint position and vibration sense at the distal interphalangeal joint, and by static two point discrimination of the palmar aspect of the distal phalanx using the Mackinnon-Dellon discriminator. ${ }^{30}$ Only two of the

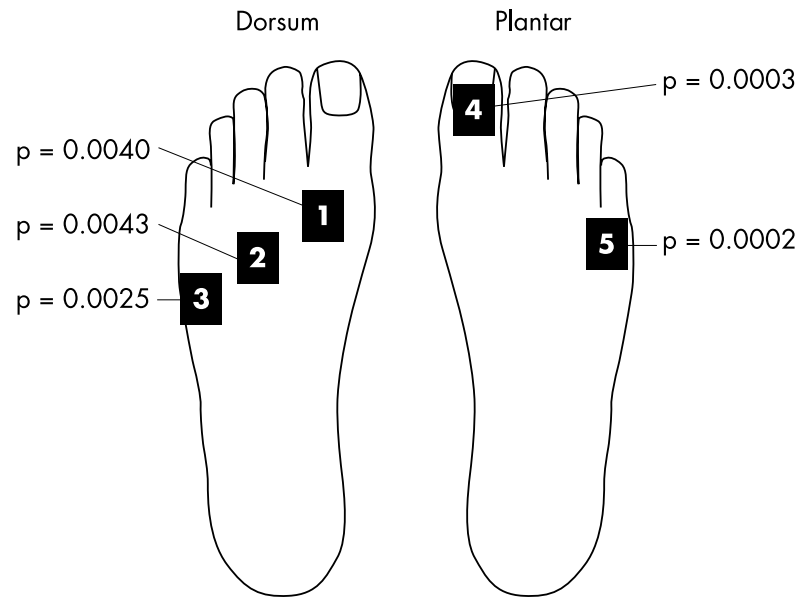

Figure 1 Dorsal and ventral foot zones tested with

Semmes-Weinstein monofilaments. The $p$ values indicate a significant difference between neuropathy and control subjects for each of the five foot zones. 
Table 2 Clinical sensation, number of limbs in each category

\begin{tabular}{|c|c|c|c|c|c|c|c|c|}
\hline & \multicolumn{4}{|c|}{ Neuropathy subjects $(n=8)$} & \multicolumn{4}{|c|}{ Control subjects $(n=10)$} \\
\hline & Normal & Reduced & Absent & No. limbs & Normal & Reduced & Absent & No. limbs \\
\hline \multicolumn{9}{|l|}{ Toe } \\
\hline Proprioception & - & 15 & 1 & 16 & 18 & 2 & - & 20 \\
\hline Vibration & - & 10 & 6 & 16 & 9 & 11 & - & 20 \\
\hline Ankle & & & & & & & - & \\
\hline Proprioception & 10 & 6 & - & 16 & 18 & 2 & - & 20 \\
\hline Vibration & 1 & 15 & - & 16 & 18 & 2 & - & 20 \\
\hline \multicolumn{9}{|l|}{ Superficial sensation } \\
\hline Dorsal feet & 4 & 12 & - & 16 & 16 & 4 & - & 20 \\
\hline Plantar feet & 2 & 14 & - & 16 & 18 & 2 & - & 20 \\
\hline \multicolumn{9}{|l|}{ Right index finger } \\
\hline Proprioception & 7 & 1 & - & 8 & 10 & - & - & 10 \\
\hline Vibration & 5 & 3 & - & 8 & 10 & - & - & 10 \\
\hline 2 point discrimination & 6 & 2 & - & 8 & 10 & - & - & 10 \\
\hline
\end{tabular}

DM-PN patients, and none of the controls had reduced index finger somatosensory sensation. For two point discrimination, age adjusted norms were applied; that is, $4 \mathrm{~mm}$ distance between the two points was established as upper normal limit. ${ }^{31-33}$ All subjects had normal joint position and vibration sense. Table 2 summarises results of the clinical examination.

\section{Experimental protocol}

Subjects were tested while standing barefoot with their eyes closed on the surface of a dual force platform. A touch plate was positioned at the subjects' right side at $90 \mathrm{~cm}$ height from the floor, immediately anterior to the right foot. Each subject was tested under three touch conditions-no touch (NT), light touch (LT), and heavy touch (HT) - during three backward translation velocities of 10,20, and $30 \mathrm{~cm} / \mathrm{s}$. Translation amplitude was kept constant at $6 \mathrm{~cm}$. In the NT condition the right hand was held above the touch bar surface, keeping a distance of at least $10 \mathrm{~cm}$ between the plate and the index finger. During LT trials, the plate was contacted by the pulp of the right index finger with a pressure not exceeding $1 \mathrm{~N}$. An audiofeedback device informed subjects when $1 \mathrm{~N}$ vertical force on the touch plate was accidentally exceeded. During tests with HT, subjects were allowed to use the touch plate for mechanical support by transmitting onto it as much force as they chose with their index finger. Altogether, each subject underwent 45 trials given in five-trial blocks in nine randomly presented conditions. A safety harness was used to minimise fall risks.

During the experiment, rectified EMG data (bandwidth: $70-2000 \mathrm{~Hz}$ ) were sampled at a frequency of $480 \mathrm{~Hz}$ for eight seconds, with a two second pre-perturbation baseline period. EMG signals were low passed filtered with a cut off frequency of $100 \mathrm{~Hz}$. Vertical and horizontal forces transmitted both onto the touch plate and onto the force platforms under the feet were sampled at $240 \mathrm{~Hz}$. Whole body centre of pressure (CoP) in the anterior-posterior (AP) and medio-lateral (ML) directions were calculated from four vertical and two horizontal force transducers in each foot plate.

\section{Data analysis}

Three outcome variables were used for analysis: (1) EMG response latencies of the two medial gastrocnemius (MG) muscles; (2) total body CoP response latencies; and (3) initial (first $75 \mathrm{~ms}$ ) active rate of change of $\mathrm{CoP}$ (CoP velocity) in the AP and ML directions. The first $75 \mathrm{~ms}$ represents the initial automatic postural response prior to voluntary intervention. Initial postural EMG responses have a duration of $75 \mathrm{~ms}$ that, together with their resulting initial rate of change in CoP, are linearly scaled to stimulus velocities based on somatosensory feedback but not influenced by predicted stimulus characteristics. $^{34}{ }^{35}$ Response latencies were determined by an interactive computer program as the time at which the level of the MG EMG activity or of the CoP displacement exceeded its mean baseline value by 2 standard deviations for at least 75 ms. Descriptive statistics and 2 (groups) $\times 3$ (touch conditions, repeated measures) $\times 3$ (velocity conditions, responsive measures) ANOVAs were used to study the differences between neuropathy and control subjects and their interactions.

\section{RESULTS}

\section{Gastrocnemius response latencies}

Response latencies of the left and right medial gastrocnemius muscles to backward movement of the platform (paired $t$ test) were not different and therefore their results were combined. The response latencies of the patients were longer than responses of the healthy subjects despite velocity of displacement or touch condition $(\mathrm{F}=[1,16] 5.5 ; \mathrm{p}=0.02)$ with a mean delay of 6.5 milliseconds in patients' responses. Figure 2 presents representative EMG recordings of the right MG muscle of one control subject and of one neuropathy patient at platform velocity of $30 \mathrm{~cm} / \mathrm{s}$. Despite the longer than normal gastrocnemius response latencies, fingertip touch did not shorten latencies in neuropathy subjects. There was no significant effect of light or heavy touch, no significant effect

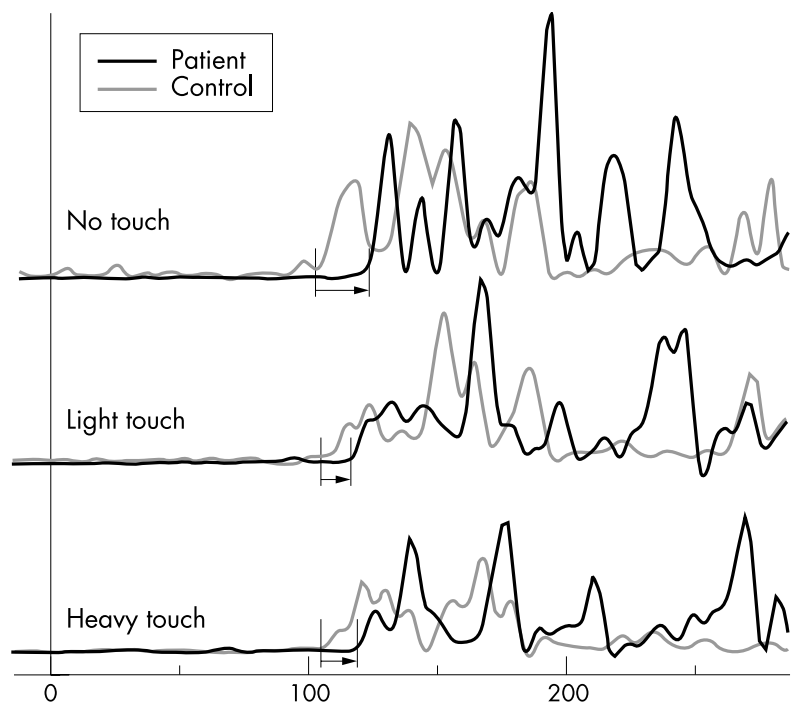

Figure 2 Raw EMG recordings from the right medial gastrocnemius muscle of one control subject (grey line) and one neuropathy subject (black line), for each of the touch conditions. EMG onset occurred later in the neuropathy subject. Time on the $x$ axis is in $m s$. 

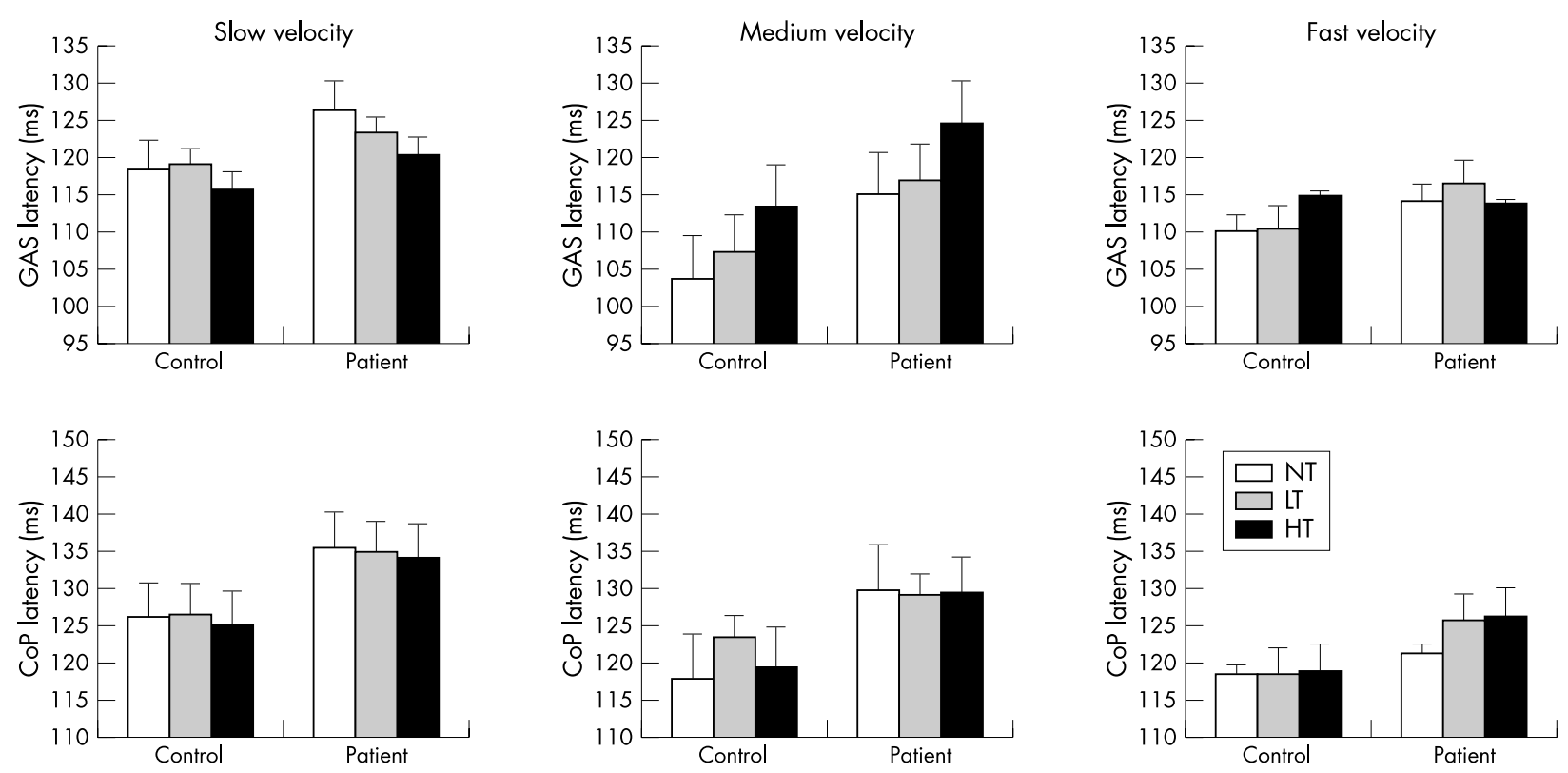

Figure 3 Mean response latencies of the medial gastrocnemius muscle to three different velocities of posterior platform translations and three touch conditions (top row). Mean anterior-posterior CoP latencies in response to three velocities of posterior platform perturbations and three touch conditions (bottom row).

of surface translation velocity, and no interaction effect on gastrocnemius latency (fig 3). In fact, during medium velocity (but not slow or fast velocity) translations, light and heavy touch tended to increase, rather than decrease gastrocnemius response latencies in both groups, although this velocity by touch condition interaction effect was not significant at the $\mathrm{p}=0.05$ level.

\section{CoP response latencies}

Latencies of the initial active CoP response to backward platform translation were also significantly longer in the patients than in the control subjects $(\mathrm{F}=[1,16] 14.0 ; \mathrm{p}<0.001)$ with a mean difference of 8.0 ms. Figure 3 shows mean values of CoP latency for the two groups under the three touch modes and under the three platform velocities. Touch mode had no significant or consistent effect on AP CoP latency. CoP latency was, however, affected by the velocity of the platform $(\mathrm{F}=$
$[2,32] 5.54 ; p=0.02)$, so that in both groups the highest platform velocities yielded the shortest latencies and vice versa.

Scaling of initial postural response to platform velocity. The slope of the relation between the initial AP CoP velocity due to a plantar flexion response and platform translation velocity was significantly lower in patients than in the control subjects $(F=[1,16] 5.8 ; p=0.02)$. Figure 4 depicts the mean slopes of the two groups under the three touch modes. Light touch and heavy touch significantly increased the slope of scaling postural responses to platform translation velocity for both groups. The touch effect was significant $(\mathrm{F}=[2,32] 6.1$; $\mathrm{p}=0.004)$, with no significant interaction with the effect of group. However, subjects with neuropathy increased the sensitivity of response scaling with heavy, but not light touch, whereas control subjects also showed steeper slopes during light touch (post hoc tests).
Neuropathy subjects

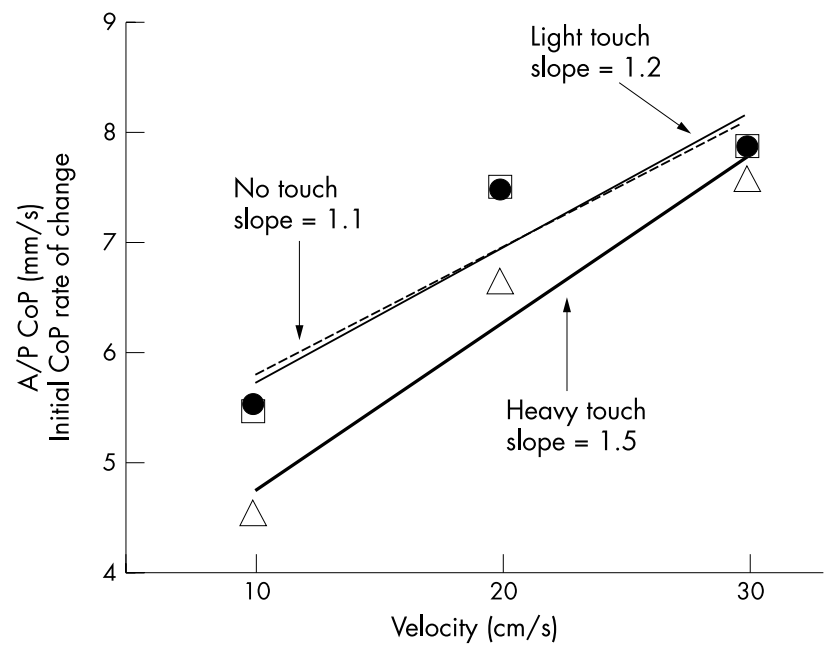

Control subjects

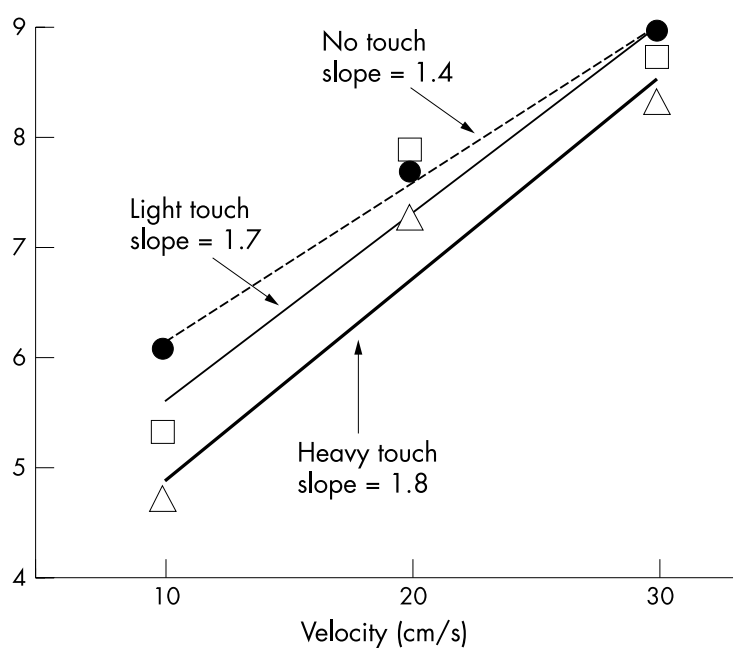

Figure 4 Scaling of initial AP CoP velocity to platform velocity during each of the three touch conditions for neuropathy and control subjects. The regression lines for each touch condition are given with the slope values. Circle, no touch; square, light touch; triangle, heavy touch. 
Neuropathy subjects

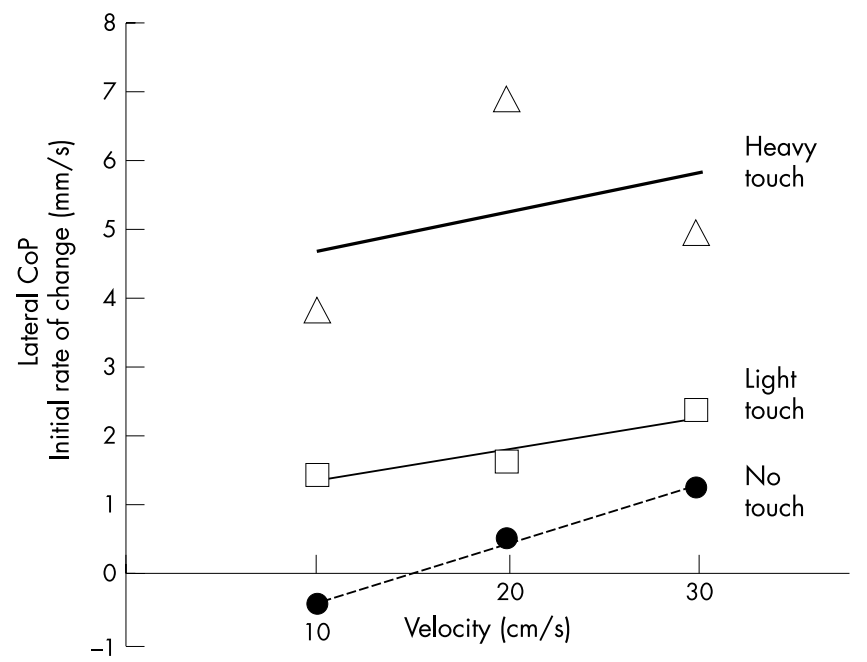

Control subjects

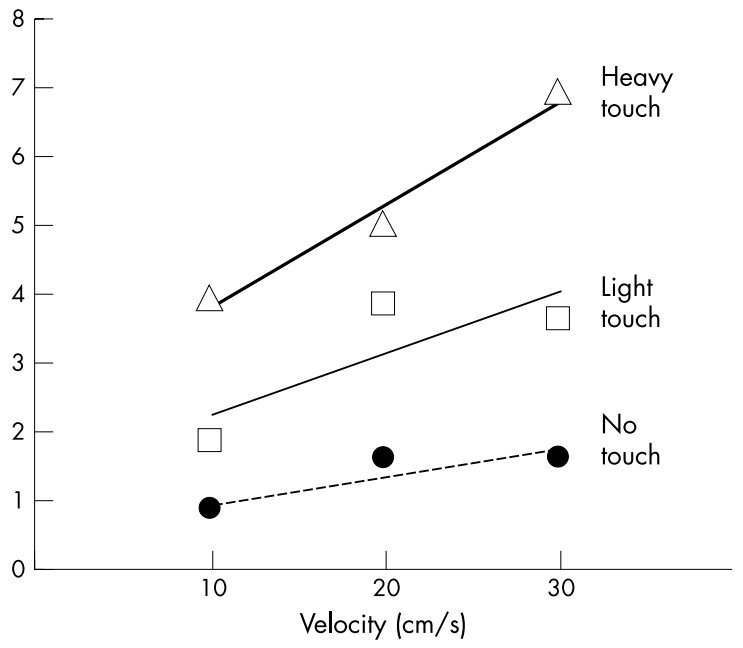

Figure 5 Scaling of initial medial-lateral CoP velocity to platform velocity during each of the three touch conditions for neuropathy and control subjects. The regression lines for each touch condition are given with the slope values. Circle, no touch; square, light touch; triangle, heavy touch.

Mean AP CoP velocity was not different between groups, although it was significantly affected by touch mode ( $\mathrm{F}=$ $[2,32] 6.1 ; p=0.004)$. Initial postural response magnitude, as quantified by CoP velocity, was lowest during the HT and highest with NT $(\mathrm{F}=3.5 ; \mathrm{p}=0.04)$. In the control subjects, touch mode effects were more pronounced than in the patients due to a smaller initial CoP velocity in LT than NT for slow platform velocities (fig 4).

\section{Lateral CoP initial velocity}

The direction of the CoP vector in response to backward surface translations was diagonally forwards and towards the right side (cane side), especially during light and heavy touch in both groups, and its magnitude tended to be larger in the control subjects than in the patients. However, this between group difference, despite its consistency in eight of the nine tested conditions did not reach the 0.05 level of significance. Mean lateral CoP values of the control subjects and the patients in the three touch conditions are presented in fig 5. Touch mode effect was significant $(\mathrm{F}=[1,16]$ 24.5; $\mathrm{p}<0.0001$ ), with the largest rightward CoP velocity in the HT mode and the lowest in the NT mode. As is obvious from fig 5 , the effect of LT in augmenting rightward CoP velocity was substantial in both groups, indicating the neuropathy subjects' ability to alter the direction of their corrective CoP responses when touching a stable surface in a similar way to healthy subjects. Rightward lateral CoP velocity was also directly related to platform velocity for backward translations; the largest rightward $\mathrm{CoP}$ velocity in response to the largest platform velocity of $30 \mathrm{~cm} / \mathrm{s}$, and the lowest in response to the lowest platform velocity of $10 \mathrm{~cm} / \mathrm{s}$.

\section{DISCUSSION}

Consistent with previous studies, the subjects with somatosensory loss from diabetic neuropathy show delayed postural response latencies (both EMG and surface forces) and reduced magnitude scaling for increasing translation velocities. ${ }^{18}$ Neither light touch nor heavy touch of a fingertip on a stable support shortened the latencies of automatic postural responses. However, scaling postural response magnitude to the velocity of postural perturbation was improved with fingertip touch, although subjects with neuropathy required heavy touch, whereas control subjects also benefited from light touch. Moreover, light touch also had two unexpected effects on postural responses: touch reduced the magnitude of the initial AP CoP responses, and increased the ML CoP responses towards the side of touch.

It has been claimed that light fingertip touch provides cutaneous information at the fingertip, which, coupled with proprioceptive information about changes in arm configuration, signal direction and velocity of body sway to the central nervous system to allow stabilising activation of leg postural muscles during stance. ${ }^{5636}$ With light touch, fingertip forces are phase advanced with respect to support surface forces under the feet, consistent with a feedforward control of posture, whereas for heavy touch, fingertip forces are in phase with support surface forces consistent with feedback control mechanism. ${ }^{2}{ }^{17}$ The current results show that such feedforward and feedback control mechanisms can affect the earliest postural responses initiated at about $100 \mathrm{~ms}$ following an unexpected external perturbation of the floor. Both the magnitude and relative scaling of the first $75 \mathrm{~ms}$ of postural response were affected by fingertip touch in both control and neuropathy subjects. This initial postural response is thought to be triggered and not be directly under voluntary control since normal voluntary reaction time activation of the gastrocnemius muscle exceeds $180 \mathrm{~ms} .^{37}$ Although postural response magnitude was affected by fingertip touch, neither healthy nor DM-PN subjects could make use of fingertip touch to alter the latencies of postural muscle activation with their eyes closed and the backward platform velocity randomly changed between trials.

Although light fingertip touch has powerful effects on stability in quiet stance, its role in dynamic postural control is not clear. The pathways from somatosensors in the hand and arm to postural control centres in the brain stem or elsewhere in the nervous system are at least as fast or faster than from the feet and legs, and there is evidence that small perturbations to the hands can result in medium latency postural responses in the legs. ${ }^{21}$ Nevertheless, we did not observe an improvement in postural responses in neuropathy or control subjects with light touch, except some reduction in sagittal forces at the ground necessary to move the CoM back to equilibrium, and an increased sensitivity (by decreasing reactive forces in response to the lowest velocity translations but not to the fast velocity translations). The effectiveness of light touch to stabilise trunk variability as a substitute for loss of vestibular or visual information or loss of vision during quiet stance or surface oscillations suggests that touch may act as an external reference for 
earth vertical. The inability of light touch to shorten the latency or increase the magnitude of automatic postural responses to external body displacements suggest that light touch does not reliably act as a sensory trigger for postural responses. ${ }^{7} 1738$

Previous studies have shown that the magnitude of postural responses are reduced or eliminated when subjects are supported at the chest or with their hands. ${ }^{22}$ This flexibility in postural response allows body parts most appropriate for current conditions to exert forces against surfaces to regain equilibrium. The initial changes in leg postural response magnitude even occurred in anticipation of the hands coming in contact with a stable surface..$^{22}$ In the current study, we show that light touch, too small to allow mechanical support, also significantly reduces AP directed postural responses but increases laterally directed postural responses, perhaps in anticipation that the hand could be used for stabilisation.

The enhancement of rightward CoP velocity (towards the touch plate) with fingertip touch was substantial in both subject groups. Presumably, the light touch cues from the touch bar provided an environmental reference that biased postural orientation to the right side. We cannot distinguish between the possibility that the rightward diagonal direction of CoP displacement in the presence of the touch plate was due to feedforward, central set effect, or to a feedback effect including sensory cues from the arm in contact with the touch plate. The effect of light touch was more pronounced in the ML plane than in the AP plane, consistent with a plane specific effect of light touch. ${ }^{683639}$ Similar findings were documented for quiet stance by Clapp and Wing, who showed that light touch of a laterally located support (similar to ours, the touch bar was located somewhat forward to the line of the shoulders) decreases CoP sway in the AP plane.." Perhaps, light touch would have suppressed, rather than enhanced ML CoP, and enhanced, rather than suppressed AP CoP if the perturbation was laterally directed, instead of backwards. By decreasing the necessary postural response to return equilibrium in the plane of perturbation, postural responses become more energy efficient, and by increasing postural response in the orthogonal plane, the hand in contact with the support surface becomes a potential "third leg" for a larger base of support. The rightward augmentation of lateral CoP was just as powerful for neuropathy subjects and control subjects, indicating the patients' ability to modify their postural responses in order to orient to the side of the supportive device.

For all other postural variables, except ML CoP velocity, LT cues were less effective in the DM-PN patients than in the control subjects. For example, scaling of AP CoP velocity to the movement velocity of the platform, improved with LT only in the control subjects, while the DM-PN patients did not sufficiently use LT for adjusting their response velocity to the velocity of the perturbation. Altogether, the results indicate that DM-PN patients could use LT for modifying the earliest automatic postural response to surface perturbation, although they benefited from it less than the healthy subjects. One explanation for the relative insensitivity of neuropathy subjects to LT is a subclinical reduction of hand cutaneous perception, ${ }^{41}$ undetectable by the routine sensory evaluations of the fingers. Perhaps, the diabetic subjects in our study fail to make optimal advantage of LT in functional postural tasks for the same reason that diabetic patients have difficulty learning Braille, ${ }^{42}$ because of subtle sensory delays from the hand. It is also possible that patients with chronic neuropathy have learned to adapt to the instability resulting from their neuropathy across the progression of their disease such that they do not profit as much as control subjects from a sensory substitution device.

Nevertheless, these results and previous studies on increased stability with light touch during quiet stance, ${ }^{6}{ }^{17}$ support the use of canes to improve postural stability and prevent falls in subjects with peripheral neuropathy of the feet. In addition to indications for their mechanical support, ${ }^{43}$ the current results show that even light, non-mechanically stabilising touch of a lateral support can improve the quality of dynamic postural responses to slips or trips.

\section{ACKNOWLEDGEMENTS}

This work was supported by NIH grants ROl-AG 06457 and ROl-DC 01849.

Authors' affiliations

R Dickstein, Dept of Physical Therapy, Tel Aviv University, Tel Aviv, Israel

R J Peterka, F B Horak, Neurological Sciences Institute of Oregon Health \& Science University, Portland, Oregon, USA

\section{REFERENCES}

1 Horak FB, Nashner LM, Diener HC. Postural strategies associated with somatosensory and vestibular loss. Exp Brain Res 1990;82:167-77.

2 Kavounoudias A, Roll R, Roll JP. The plantar sole is a "dynamometric map" for human balance control. Neuroreport 1998;9:3247-52.

3 Wu G, Zhao W. The role of mechanoreceptive information in the stability of human upright posture. A theoretical consideration. Motor Control 1997; 1 .

4 Holden M, Ventura J, Lackner JR. Stabilization of posture by precision contact of the index finger. J Vestibular Research 1994:4:285-301.

5 Jeka JJ, Lackner JR. Fingertip contact influences human postural control. Exp Brain Res 1994;100:495-502

6 Jeka JJ. Light touch contact as a balance aid. Phys Ther 1997;77:476-87.

7 Lackner JR, Dizio P, Jeka JJ, et al. Precision contact of the fingertip produces postural sway of individuals with bilateral vestibular loss. Exp Brain Res 1999;126:459-66.

8 Jeka JJ, Easton RD, Bentzen BL, et al. Haptic cues for orientation and postural control in sighted and blind individuals. Percept Psychophys 1996:58:409-23.

9 Lackner JR, Dizio P, Jeka JJ, et al. Fingertip contact suppresses the destabilizing effect of leg muscle vibration. Society for Neuroscience Abstracts, Vol. 22. Washington, 1966.

10 DiZio P, Lackner JR, Rabin E. Sensorimotor and environmental conditions constraining use of the hand as a guide for posture. 13th International Symposium: Multisensory Control of Posture \& Gait, Paris, June, 1997

11 Adler AI, Boyko EJ, Ahroni JH, et al. Risk factors for diabetic peripheral sensory neuropathy. Results of the Seattle Prospective Diabetic Foot Study. Diabetes Care 1997:20:1162-7.

12 Green DA, Sima AAF, Albers JW, et al. Diabetic neuropathy. In: Rifkin $\mathrm{H}$, Porte D, eds. Diabetes mellitus. Theory and practice. New York: Elsevier, 1999:710-53

13 Cavanagh PR, Simoneau GG, Ulbrecht JS. Ulceration, unsteadiness and uncertainty: the biomechanical consequences of diabetes mellitus. J Biomechanics Suppl 1 1993;26:23-40.

14 Giacomoni PG, Bruno E, Monticone G, et al. Postural rearrangement in IDDM patients with peripheral neuropathy. Diabetes Care 1996;19:372-4.

15 Simoneau GG, Ulbrecht JS, Derr JA, et al. Postural instability in patients with diabetic sensory neuropathy. Diabetes Care 1994;17:1121-41.

16 Boucher P, Teasdale N, Courtemanche R, et al. Postural stability in diabetic polyneuropathy. Diabetes Care 1995;18:638-44.

17 Dickstein R, Shupert CL, Horak F. Fingertip touch improves postural stability in patients with peripheral neuropathy. Gait \& Posture $2001 ; 14: 238-47$

18 Inglis JT, Horak FB, Shupert CL, et al. The importance of somatosensory information in triggering and scaling automatic postural responses in humans. Exp Brain Res 1994;101:159-64.

19 Cavanagh PR, Derr JA, Ulbercht JS, et al. Problems with gait and posture in neuropathic patients with insulin dependent diabetes mellitus. Diabetes Medicine 1992;9:469-74.

20 Katoulis EC, Ebdon-Parry M, Lanshammar H, et al. Gait abnormalities in diabetic neuropathy. Diabetes Care 1997;20:1904-7.

21 Marsden CD, Merton PA, Morton HB. Rapid postural reacitons to mechanical displacemnt of the hand in man. Adv Neurol 1983;39:645-59.

22 Nardone A, Corra T, Schieppati M. Different activation of the soleus and gastrocnemii muscles in response to various types of stance perturbation in man. Exp Brain Res 1990:80:323-32.

23 Elger, K, Wing, A, Gilles, M. Integration of the hand in postural reactions to sustained sideways force at the pelvis. Exp Brain Res 1999; 128:52-60

24 Nutt JG, Horak F. Gait and balance disorders. In: Watts R, Koller W, eds. Movement disorders. Neurologic principles and practice. McGraw-Hill, 1995

25 Richardson JK, Hurvitz EA. Peripheral neuropathy: a true risk factor for falls. J Gerontol 1995;50:M211-15.

26 Richardson JK, Ashton-Miller JA. Peripheral neuropathy: an often-overlooked cause of falls in the elderly. Postgrad Med 1996;99:161-72. 
27 Woolacott $\mathrm{MH}$. Age related changes in posture and movement. J Gerontol 1993;48:56-60

28 Bell JA. Semmes Weinstein monofilaments testing for determining cutaneous light touch/deep pressure sensation. The Star/Carville 1984

29 Holewski JJ, Stess RMGP, Grunfeld C. Aesthesiometry: quantification of cutaneous pressure sensation in diabetic peripheral neuropathy. J Rehabil Res Dev 1988;25: 1-10.

30 Mackinnon SE, Dellon AL. Two point discrimination tester. J Hand Surg (Am) 1985:906-7.

31 Desroisiers J, Hebert R, Bravo G, et al. Hand sensibility of healthy older people. J Am Geriatr Soc 1996;44:974-8.

32 Louis DS, Green TL, Jacobson KE, et al. Evaluation of normal values for stationary and moving two point discrimination in the hand. J Hand Surg (Am) 1984;9A:552-5.

33 Dyck PJ, Dyck PJB, Bravo G, et al. Ten steps in characterizing and diagnosing patients with peripheral neuropathy. Neurology 1996:47:10-17.

34 Diener HC, Horak, FB, Nashner LM. Influence of stimulus parameters on human postural responses. J Neurophysiol 1988;59:1888-905

35 Horak FB, Diener, HC. Cerebellar control of postural scaling and central set in stance. J Neurophysiol 1994;72:1-15.
36 Jeka JJ, Lackner JR. The role of haptic cues from rough and slippery surfaces in human postural control. Exp Brain Res 1995;103:267-76.

37 Nashner LM, Cordo PJ. Relation of automatic postural responses and reaction time voluntary movements of human leg muscles. Exp Brain Res 1981;43:395-405.

38 Creath $\mathbf{R}$, Kiemel T, Horak FB, et al. Limited control strategies with the loss of vestibular function. Exp Brain Res 2002:145:323-33.

39 Nandapalan V, Smith CA, Jones AS, et al. Objective measurement of the benefit of walking sticks in peripheral vestibular balance disorders using the Sway Weigh balance platform. J Laryngol Otol 1995; 109:836-40

40 Clapp SC, Wing AM. Light touch contribution to balance in normal bipedal stance. Exp Brain Res 1999;1 25:521-4

41 Mackel R. Properties of cutaneous afferents in diabetic neuropathy. Brain 1989;112:1359-76.

42 Heinrichs RW, Moorhouse JA. Touch perception thresholds in blind diabetic subjects in relation to reading the Braille type. N Engl J Med 1969:280:72-5.

43 Ashton-Miller JA, Yeh MW, Richardson JK, et al. A cane reduces loss of balance in patients with peripheral neuropathy: results from a challenging unipedal balance test. Arch Phys Med Rehabil 1996:77:446-52.

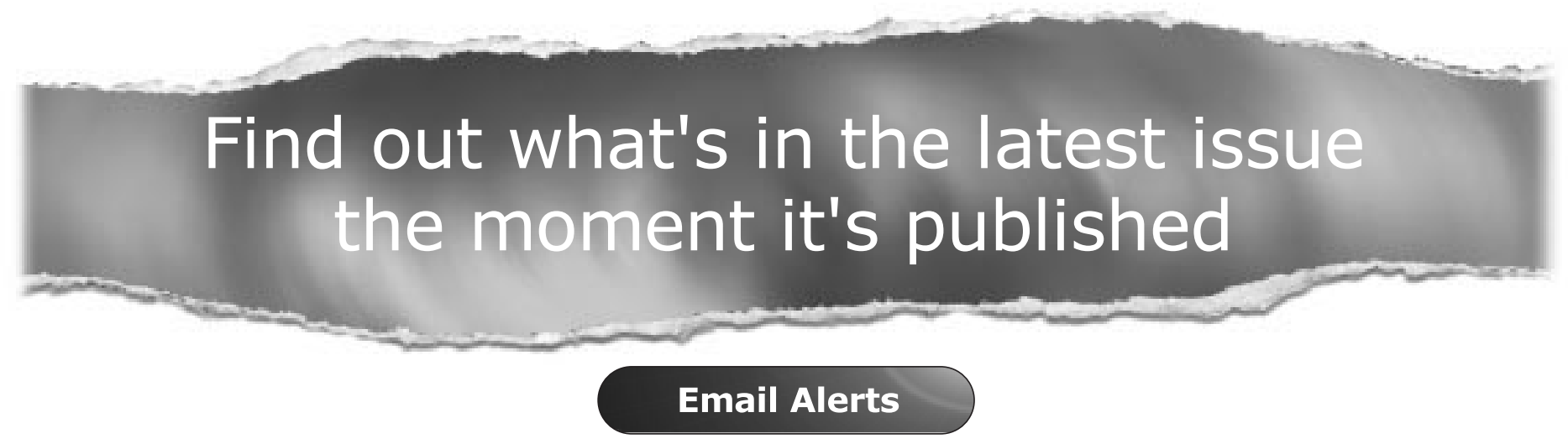

Sign up to receive the table of contents by email every month. You can select from three alerts: Table of Contents (full), TOC Awareness (notice only); Journal of Neurology, Neurosurgery, and Psychiatry related announcements. 